\title{
Relationship Between Burst Properties and Sensitivity to Input: A Theoretical Analysis
}

\author{
E. SIVAN \\ Department of Neurobiology and Department of Computer Science, Hebrew University, Jerusalem 91904, Israel \\ ehuds@cs.huji.ac.il \\ H. PARNAS \\ Department of Neurobiology and the Center for Neural Computation, Hebrew University, Jerusalem 91904, Israel \\ D. DOLEV \\ Department of Computer Science, Hebrew University, Jerusalem 91904, Israel
}

Received August 1, 1994; Revised August 3, 1995; Accepted August 18, 1995

Action Editor: Eve Marder

\begin{abstract}
This paper examines the sensitivity of endogenous bursters to a brief input pulse. The interneurons of the lobster cardiac ganglion were selected as a case study.

Using a mathematical model specifically developed for the neurons in the cardiac ganglion of the lobster (Av-Ron et al., 1993), we show a tight link between burst characteristics and certain other parameters. We show that cells with different burst properties differ in their sensitivity to an input of a brief pulse.

Irrespective of these differences, all cells display a bimodal response to a brief pulse applied during the quiescent period. During the first three-quarters of the quiescent period, they respond by producing a single spike at most. During the remaining one-quarter, the brief pulse can initiate the cells' intrinsic burst. Our predictions fit experimental results obtained by Tazaki and Cooke (1979).

The results obtained herein are discussed with respect to fault tolcrance considerations.
\end{abstract}

Keywords: fault tolerance, sensitivity, cardiac ganglion, endogenous burster

\section{Introduction}

This paper is the first in a series of studies that explores possible mechanisms of fault tolerance in small neuronal networks. Fault tolerance in biological systems can be ideally studied in the cardiac ganglion of the lobster because this ganglion is vital to the existence of the organism.

Fault tolerance of a network must certainly be influenced by the intrinsic properties of the individual cells in the network and by their response to input. We, therefore, start our long-range project by investigating these two aspects.

We focus on the lobster Panulirus interuptus, as the bursting behavior of its interneurons has been analyzed in some detail (Friesen, 1975a, b). The cardiac ganglion consists of four interneurons and five motoneurons. All four interneurons exhibit bursting behavior but differ in two of the three main characteristics of a burst; all have the same cycle duration, but they differ in both the frequency and the duration of the spiking period (Friesen, 1975a). 
The first step in our theoretical analysis was to extend the work of Av-Ron (1992) by constructing mathematical "model cells" that exhibit the biologically observed bursting characteristics. In the process of constructing these model cells, we could show a close correlation between a certain of parameters and the various modes of bursting behavior. Having modeled the intrinsic properties of the various interneurons, we next examined the effect of input on their behavior. We naturally started with the most elementary input, a brief depolarizing pulse. While model cells with different burst properties show different sensitivity to input, all model cells display a bimodal response to a brief pulse applied during the quiescent period. During the first three-quarters of the quiescent period, they respond by producing a single spike at most. During the remaining one-quarter, the brief pulse can initiate the cells' intrinsic burst. These results resemble the experimentally observed response (Tazaki and Cooke, 1979).

\section{Biological Background}

In the cardiac ganglion of the lobster Panulirus interuptus the five motoneurons (labeled 1 to 5 see schematic diagram in Fig. 1) innervate the heart muscle directly and the four interneurons (labeled 6 to 9 see diagram in Fig. 1) innervate each other and the motoneurons. These cells control the cycle of the ganglion and, thus, the heart rate. Cell 9 is the first to fire in the ganglionic spiking period, cell 8 follows and then cells 7 and 6 . Cell 6 is the main interneuron that drives the motoneurons, which fire only during cell 6's spiking period. The ganglionic spiking period ends in the reversed order; cell 6 stops first, followed by cell 7 , then cell 8 and finally cell 9 (Friesen, 1975a, b).

Figure 1 depicts the firing frequency of the individual interneurons during the ganglionic spiking period. Cell 9 exhibits the longest spiking period with the lowest frequency. Cell 6 , on the other hand, has
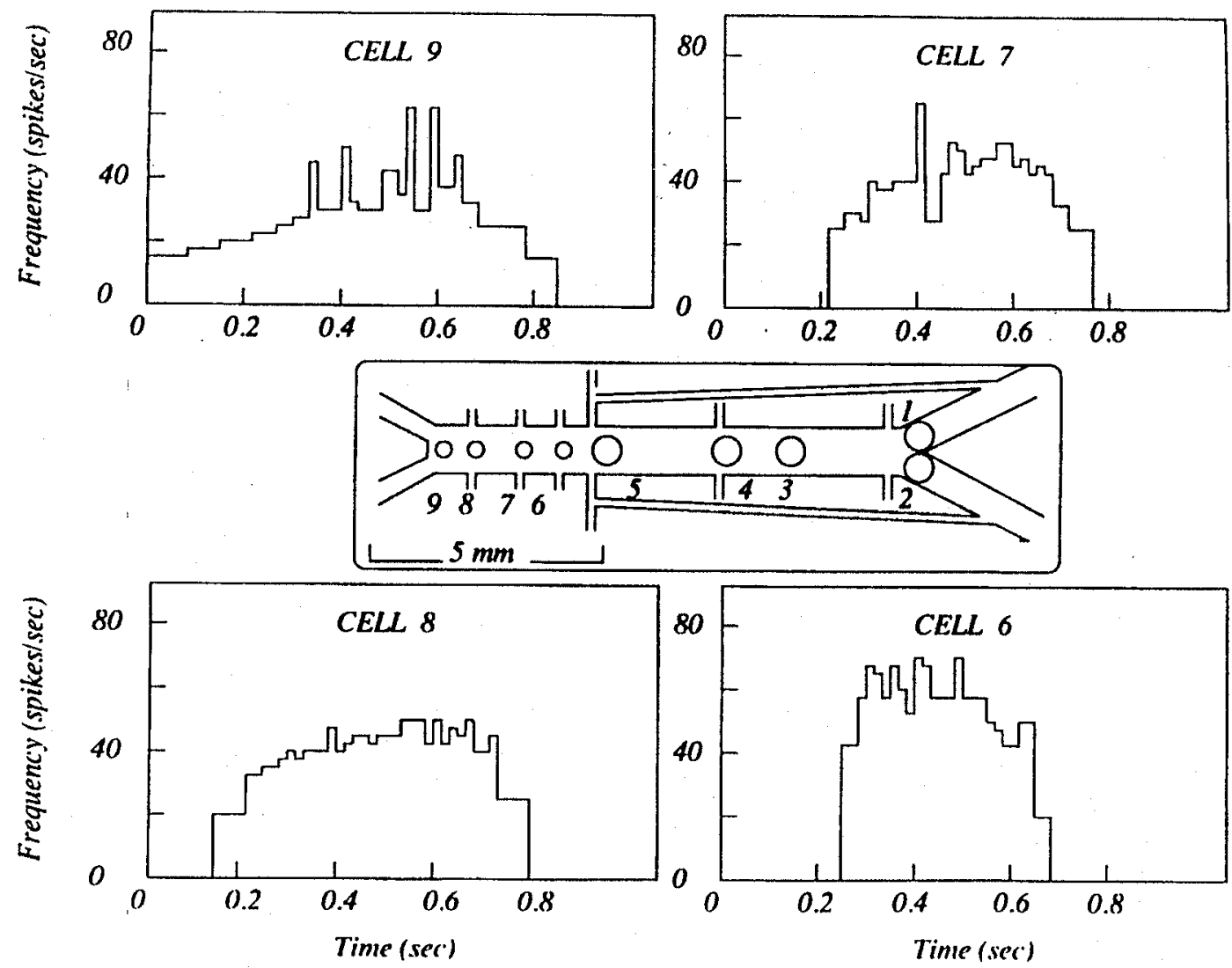

Figure 1. Frequency of firing of the interneurons: Experimental results. The instantaneous firing rate of each interneuron is plotted against time. Note the lower average firing rate of cell 9 and its relatively long duration in comparison to cell 6 (redrawn from Friesen, $1975 a$ ). Inset: Schematic representation of the cardiac ganglion of the lobster Panulirus interuptus showing the number and the location of the cell bodies. Cells 1 to 5, the large cells, are motoneurons innervating the heart. Cells 6 to 9 , the small cells, are interneurons (redrawn from Friesen, 1975a). 
the shortest spiking period and the highest frequency. Cells 7 and 8 have intermediate frequencies and spiking periods. Cell 9 was shown experimentally to be an endogenous burster (Friesen, 1975b; Hartline, 1967). Based on theoretical considerations, Av-Ron (1992) argued that the other three interneurons are also probably endogenous bursters.

Imperative to our study is that the mathematical model describing the neurons is based as much as possible on experimental data. Also, the outputs of the mathematical "model cells" must replicate the observed biological behavior. With some minor modification (justifications are given in the text), we use the mathematical model specifically developed for the interneurons of the cardiac ganglion of the lobster by Av-Ron et al. (1993). Since the mechanism underlying bursting of the interneurons is not known, Av-Ron et al. (1993) selected a mechanism that was both biologically reasonable and as simple and general as possible.

\section{The Mathematical Model Cells}

The full model for a model cell is

$$
\begin{aligned}
C_{m} \frac{d V}{d t}= & I_{\mathrm{app}}-I_{\mathrm{Na}}(W, V)-I_{\mathrm{K}}(W, V)-I_{L}(V) \\
& -I_{\mathrm{K}(\mathrm{Ca})}(\mathrm{Ca}, V)-I_{\mathrm{Ca}}(X, V)
\end{aligned}
$$

In describing the currents, we follow a now-standard approximation scheme (Rinzel, 1984). Thus,

$$
\begin{aligned}
I_{\mathrm{Na}}(W, V) & =\bar{g}_{\mathrm{Na}} m_{\infty}^{3}(V)(1-W)\left(V-V_{\mathrm{Na}}\right), \\
I_{\mathrm{K}}(W, V) & =\bar{g}_{\mathrm{K}}\left(\frac{W}{s}\right)^{4}\left(V-V_{\mathrm{K}}\right) \\
I_{L}(V) & =\bar{g}_{L}\left(V-V_{L}\right)
\end{aligned}
$$

where, $\vec{g}_{\mathrm{Na}}, \bar{g}_{\mathrm{K}}$ and $\bar{g}_{L}$ are the corresponding ionic maximal conductances, while $V_{\mathrm{Na}}, V_{\mathrm{K}}$ and $V_{L}$, denote the reversal potentials of the corresponding currents. In (2), the steady-state sodium activation gate, $m_{\infty}(V)$, is described by a general sigmoid function

$$
F_{\infty}\left(V ; a, V_{1 / 2}\right)=\frac{1}{1+e^{-2 a\left(V-V_{1 / 2}\right)}},
$$

where $a$ controls the sigmoid's steepness and $V_{1 / 2}$, its midpoint.

In this case,

$$
m_{\infty}(V)=F_{\infty}\left(V ; a_{m}, V_{m}\right)
$$

The recovery variable $W$ is described by

$$
\frac{d W}{d t}=\frac{W_{\infty}(V)-W}{\tau(V)},
$$

where the steady-state value $W_{\infty}(V)$ is modeled similarly to $m_{\infty}(V)$ by

$$
W_{\infty}(V)=F_{\infty}\left(V ; a_{W}, V_{w}\right)
$$

and the relaxation time of recovery $\tau(V)$ is given by

$$
\tau(V)=\frac{1}{\lambda\left(e^{a_{W}\left(V-V_{W}\right)}+e^{-a_{W}\left(V-V_{W}\right)}\right)} .
$$

This expression for $\tau(V)$ is based on the assumption that $W$ follows first-order kinetics for the transition from an active to an inactive state (Av-Ron et al., 1991).

The two additional currents in (1) $I_{\mathrm{Ca}}(X, V)$ and $I_{\mathrm{K}(\mathrm{Ca})}(\mathrm{Ca}, V)$ are described somewhat differently from the classical ones. The calcium current exhibits saturative dependence on extracellular $\mathrm{Ca}^{2+}$ concentration even at low concentration (Hagiwara and Takahashi, 1967; Akaike et al., 1978). In line with convention, we describe $I_{\mathrm{Ca}}(X, V)$ as the product of membrane conductance and the ion driving force:

$$
I_{\mathrm{Ca}}(X, V)=\bar{g}_{\mathrm{Ca}} X \cdot V_{\mathrm{Ca}}(V)
$$

Here, membrane conductance is accounted for by $\bar{g}_{\mathrm{Ca}}$, the maximal calcium conductance, and $X$, the fraction of open calcium channels. To model saturation, we replace the conventional driving force (which was also used by Av-Ron et al., 1993) by

$$
V_{\mathrm{Ca}}(V)=\frac{\bar{V}_{\mathrm{Ca}} C e}{C e+K e \cdot K e_{\infty}(V)},
$$

where $\vec{V}_{\mathrm{Ca}}$ is the maximal possible driving force and $\mathrm{Ce}$ is the external calcium concentration. The term $K e_{\infty}(V)$ is a sigmoid function of $V$ given as

$$
K e_{\infty}(V)=F_{\infty}\left(V ; a_{K e}, V_{K e}\right)
$$

$K e_{\infty}(V)$ increases with depolarization and represents the decreasing permeability of the channels to inward movements of calcium as membrane depolarization increases. The combined dependence of $X$ and $K e_{\infty}(V)$ on membrane potential generates the experimentally observed bell-shaped dependence of $I_{\mathrm{Ca}}$ on $V$ (Llinas 
et al., 1981). Accordingly, $X$ saturates at higher depolarizations where as $K e_{\infty}(V)$ continues to rise. As a result, $I_{\mathrm{Ca}}$ first rises due to an increase in $X$ but then declines due to a saturation of $X$ and increasing $K e_{\infty}(V)$ (for detailed a discussion see Parnas and Segel, 1989).

The third differential equation in the model describes the time course of $X$ :

$$
\frac{d X}{d t}=\frac{X_{\infty}(V)-X}{\tau_{X}},
$$

where $X_{\infty}(V)$, stands for the fraction of open channels at steady state and is described by the sigmoid curve

$$
X_{\infty}(V)=F_{\infty}\left(V, a_{X}, V_{X}\right) .
$$

The last current considered in the model, $I_{\mathrm{K}(\mathrm{Ca})}$ (Ca, $V$ ), describes a current through a potassium channel that is activated in a saturative manner by intracellular calcium, Ca. Thus,

$$
I_{\mathrm{K}(\mathrm{Ca})}(\mathrm{Ca}, V)=\bar{g}_{\mathrm{K}(\mathrm{Ca})} \frac{\mathrm{Ca}}{\mathrm{K}_{d}+\mathrm{Ca}}\left(V-V_{\mathrm{K}}\right) .
$$

The fourth differential equation in the model describes the temporal distribution of intracellular calcium concentration. The calcium concentration rises due to the voltage dependent influx of calcium (first term in 16) and declines due to a lumped removal process whose rate is depicted in the second term of (16). There, $R$ stands for the maximal rate of removal and $\mathrm{K}_{r}$ for the half-saturation constant:

$$
\frac{d \mathrm{Ca}}{d t}=Y_{\mathrm{Ca}}\left(-I_{\mathrm{Ca}}(X, V)\right)-R \frac{\mathrm{Ca}}{\mathrm{Ca}+\mathrm{K}_{r}} .
$$

Here, $I_{\mathrm{Ca}}(X, V)$ (from 10) is converted to internal concentration where $Y_{\mathrm{Ca}}$ is the translation coefficient.

In summary, the model consists of four differential equations: for $V_{1}$, the membrane potential (1); for $W$, the recovery variable (7); for $X$, the fraction of open calcium channels (13); and for $\mathrm{Ca}$, the intracellular calcium concentration (16). Of these, the first two are fast, with intrinsic time scales of milliseconds. The last two are slow, with intrinsic time scales of tens and hundreds of milliseconds respectively.

Figure 2 depicts the behavior of the four model cells ((1) to (16)). The values selected for the various parameters were based on a detailed analysis carried out by Av-Ron (1992). However, final adjustments of values aimed at (1) showing a reasonable similarity to the cells when connected to each other (see Fig. 1); (2) showing
Table 1. Parameters in which the various cells differ from each other.

\begin{tabular}{lllll}
\hline Parameter & Cell 6 & Cell 7 & Cell 8 & Cell 9 \\
\hline $\bar{g}_{\mathrm{K}(\mathrm{Ca})\left(\mathrm{mS} / \mathrm{cm}^{2}\right)}$ & 11 & 4.55 & 4.55 & 1.9 \\
$\bar{g}_{\mathrm{K}}\left(\mathrm{mS} / \mathrm{cm}^{2}\right)$ & 8 & 15 & 15 & 50 \\
$\bar{g}_{\mathrm{Ca}}\left(\mathrm{mS} / \mathrm{cm}^{2}\right)$ & 1.7 & 1.25 & 1.3 & 0.86 \\
$R(\mu \mathrm{M} / \mathrm{msec})$ & 0.0019 & 0.0012 & 0.00175 & 0.001 \\
\hline
\end{tabular}

a stable burst pattern over time (we ensured stability over $400 \mathrm{sec}$ of simulation). The network simulation was carried out with the aid of SONN, a software package developed in our laboratory (for details, see Parnas and Rudolph, 1991). The parameter values common to all model cells are provided in the legends of Fig. 2, while those in which the model cells differ from each other are summarized in Table 1.

The behavior of the model cells in Fig. 2 shows a satisfactory resemblance to that of the cells in Fig. 1; in particular, cell 9 is the first to fire and exhibits a relatively low firing frequency at the beginning of the spiking period. As in the experimental results the other three model cells join in 100 to $200 \mathrm{msec}$ later in the sequence $8,7,6$. Cell 6 is the first to stop after about $400 \mathrm{msec}$, followed by cell 7 , cell 8 , and finally cell 9 . Cell 6 shows the highest firing frequency (see both Figs. 1 and 2). Cells 7 and 8 exhibit a fairly steady rate of firing throughout the spiking period. Finally, cell 9 starts with the lowest firing rate but this rises once the other cells join in. In all four model cells, the sharp fluctuations in frequency are due to input from the other three cells. This is probably the reason for the similar fluctuations seen in Fig. 1.

In spite of the resemblance between the model cells (Fig. 2) and the cells (Fig. 1), there are still a number of differences. For example, model cell 7 has a somewhat shorter spiking period. Also, the decline in firing rate in model cell 8 is less abrupt than in cell 8 . We feel, however, that model cells 6 and 9 , on which the present study concentrates, capture the main features of the activity of the biological cells and hence are adequate for further study. We selected cells 6 and 9 to study sensitivity to input because these cells exhibit two extreme modes of bursting behavior, while cells 7 and 8 exhibit an intermediate mode.

To examine sensitivity to input, the intrinsic properties of model cells 6 and 9 must be ascertained when they are isolated rather than connected to all interneurons (Fig. 2). Figure 3 shows the bursting behavior of isolated model cells 6 and 9. Notice the low firing 

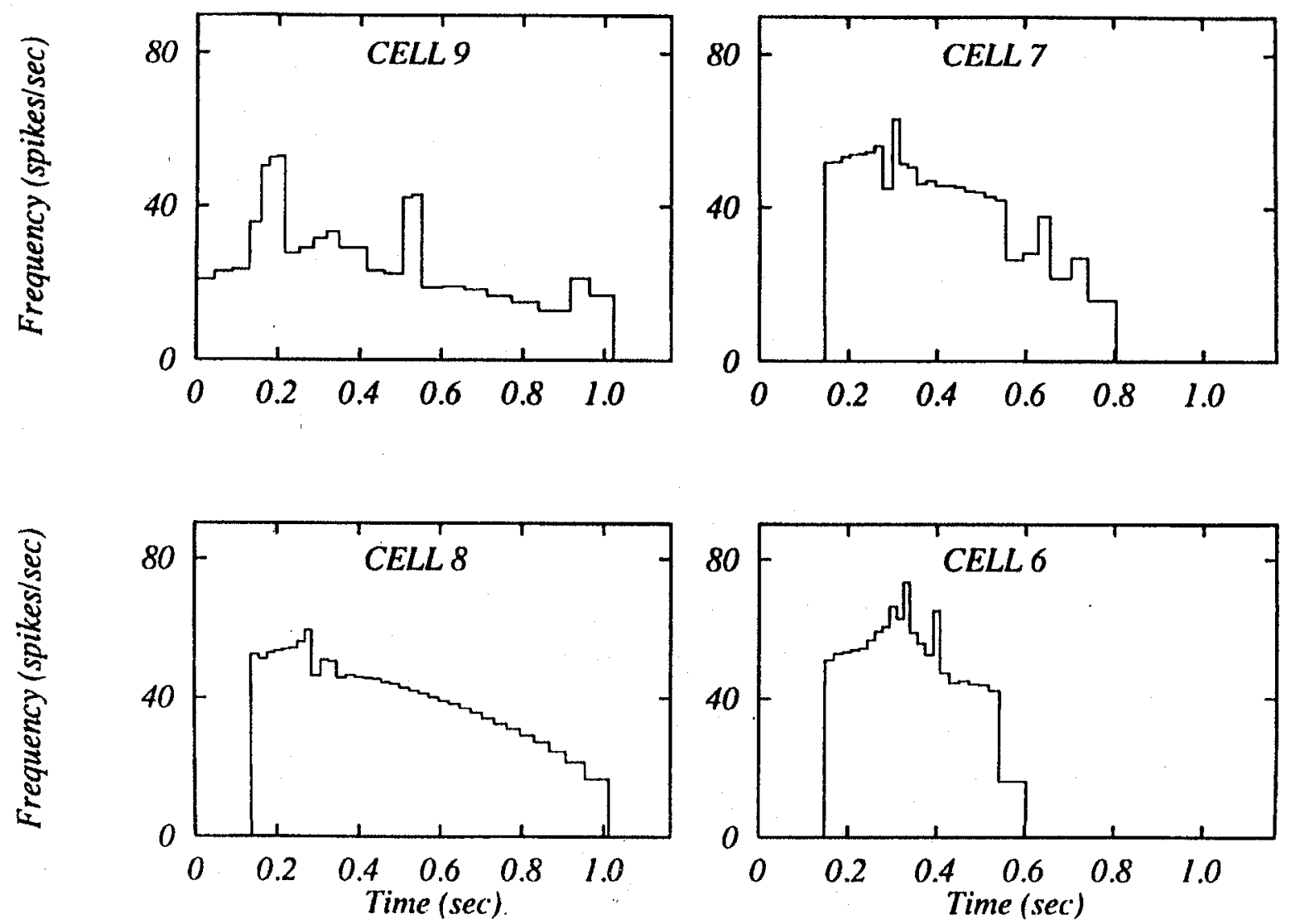

Figure 2. Frequency of firing of the model cells. The burst shown was obtained after $200 \mathrm{sec}$ of simulation to ensure stability of the results. The instantaneous firing rate is plotted as in Fig. 1. Following the presentation of Friesen (1975a) we assign zero to the beginning of the spiking period of cell 9. The values of the parameters common to the four cells are $C_{m}=1 \mu \mathrm{F} / \mathrm{cm}^{2}, \bar{g}_{\mathrm{Na}}=100, V_{\mathrm{Na}}=55, V_{\mathrm{K}}=-72, s=1, \bar{g}_{L}=0.3$, $V_{L}=-60, a_{m}=0.055, V_{m}=-30, a_{W}=0.045, V_{W}-47, \lambda=0.02, \bar{V}_{\mathrm{Ca}}=-180, C e=10 \mu \mathrm{M}, K e=100 \mu \mathrm{M}, a_{K e}=0.04, V_{K e}=60$, $a_{X}=0.18, V_{X}=-50, \tau_{X}=50 \mathrm{msec}, K_{d}=0.5 \mu \mathrm{M}, K_{r}=0.5 \mu \mathrm{M}, Y=0.00002 \mathrm{M} / \mu \mathrm{A} \cdot \mathrm{msec}$. Units: conductances $\left(\mathrm{mS} / \mathrm{cm}^{2}\right)$, voltages $(\mathrm{mV})$. The values of the parameters in which the cells differ from one another are given in Table 1. The parameter's values described here will be used in all calculations that follow.

frequency of the isolated model cell 9 (inset, Fig. 3) in comparison to its firing frequency when connected in the network (Fig. 2).

\section{Correlation Between Burst Characteristics and Model Parameters}

Can the typical behavior of either cell 6 or cell 9 be obtained by only one set of parameters or is it retained in a domain of parameters? To answer this question, we first defined group- 6 and group-9, which are variations of cells 6 and 9. To do this, we examined the burst characteristics of each cell in isolation (Fig. 3) and arbitrarily defined the burst variations around these characteristics (see schematic bursts in Fig. 4).

Next, the parameters, in which cell 6 and 9 differ (see Table 1), were systematically varied to discover how the different parameters affect the burst characteristics. We found that an increase in both $\bar{g}_{\mathrm{K}}$ and $\bar{g}_{\mathrm{K}(\mathrm{Ca})}$, decreases the cycle duration, the spiking duration, and the number of spikes. In contrast, an increase in $\bar{g}_{\mathrm{Ca}}$ increases all three characteristics. Finally, an increase in $R$ increases the spiking duration and the number of spikes but decreases the cycle duration. These results hold for a wide range of parameter values (i.e., $5 \mathrm{mS} / \mathrm{cm}^{2}<\bar{g}_{\mathrm{K}}<70 \mathrm{mS} / \mathrm{cm}^{2} ; 0 \mathrm{mS} / \mathrm{cm}^{2}<$ $\bar{g}_{\mathrm{K}(\mathrm{Ca})}<30 \mathrm{mS} / \mathrm{cm}^{2} ; 0 \mathrm{mS} / \mathrm{cm}^{2}<\bar{g}_{\mathrm{Ca}}<5 \mathrm{mS} / \mathrm{cm}^{2}$ and $0.0005 \mu \mathrm{M} / \mathrm{msec}<R<0.01 \mu \mathrm{M} / \mathrm{msec}$ ). There 

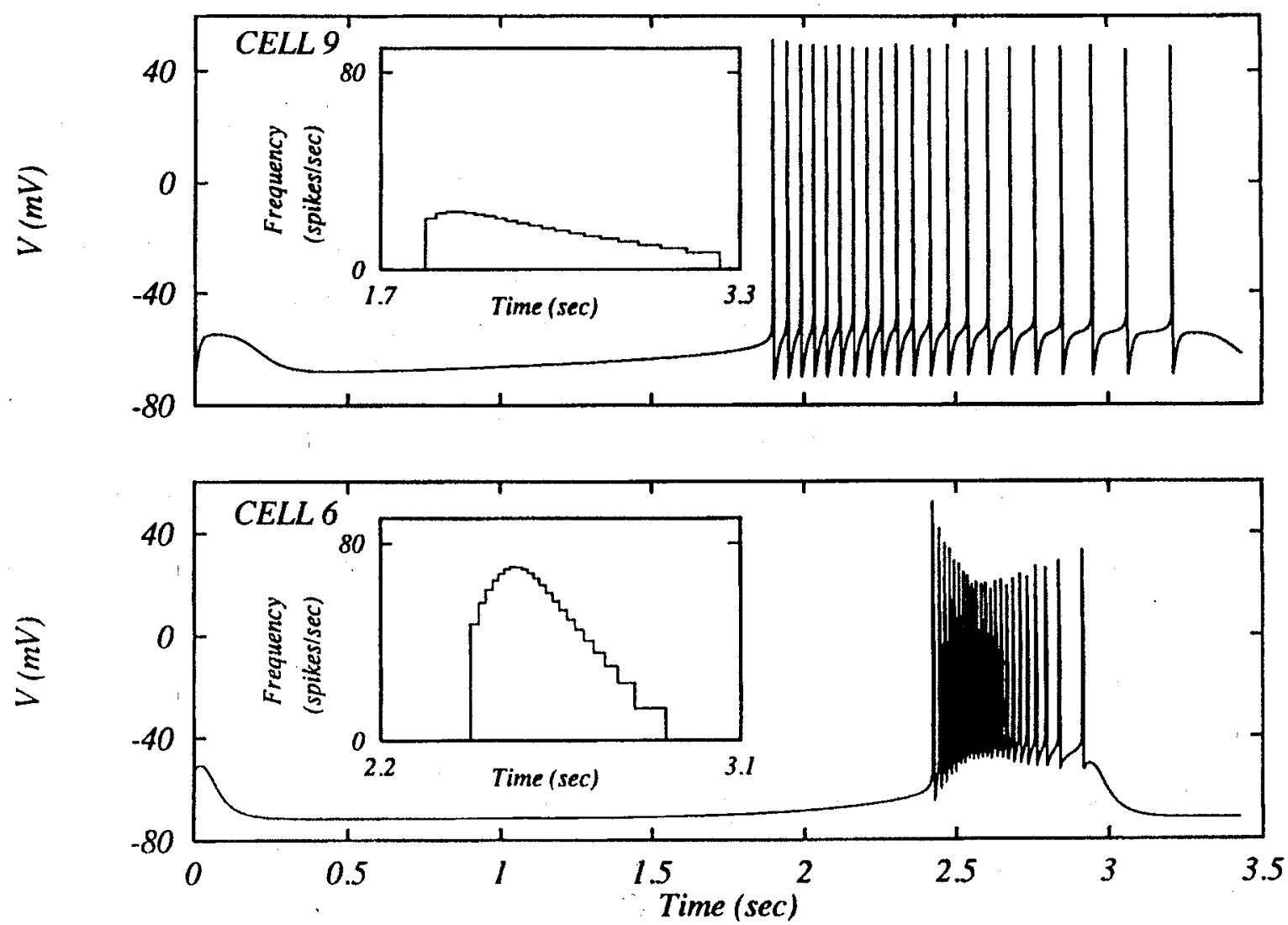

Figure 3. The temporal pattern of membrane potential in model cells 9 and 6 . The membrane voltage during one burst cycle is drawn. We arbitrarily defined $T=0$ to be at the beginning of the quiescent period. Inset: Firing frequency of isolated cell 9 (top) and cell 6 (bottom).

were some exceptions to the behavior described above. For example, for some values of $R$ in the domain of study, the cycle duration increased (rather than decreased) as $R$ increased. These cases, however, were rare and were not considered further.

The results obtained from this rather tedious search indicated that $\left(\bar{g}_{\mathrm{K}}, \bar{g}_{\mathrm{K}(\mathrm{Ca})}\right)$ plane contains two discrete areas, one where the variations of cell 6 can be found and one where the variations of cell 9 exist (Fig. 4). Furthermore, in the relevant domains, the values of the two remaining parameters, $\vec{g}_{\mathrm{Ca}}$ and $R$, can be approximated by a linear combination of the former parameters (see (17) to (20)). Thus we found (by linear regression), that for cell 6

$$
\begin{array}{r}
\bar{g}_{\mathrm{Ca}}=0.042982 \bar{g}_{\mathrm{K}}+0.122478 \bar{g}_{\mathrm{K}(\mathrm{Ca})}+0.013158 \\
(n=9, \sigma=0.070521), \\
R=0.000012 \bar{g}_{\mathrm{K}}+0.000173 \bar{g}_{\mathrm{K}(\mathrm{Ca})}-0.000022 \\
(n=9, \sigma=0.000113) .
\end{array}
$$

For cell 9 we found

$$
\begin{array}{r}
\bar{g}_{\mathrm{Ca}}=0.001159 \bar{g}_{\mathrm{K}}+0.401936 \bar{g}_{\mathrm{K}(\mathrm{Ca})}+0.010554 \\
(n=19, \sigma=0.049039), \quad(19) \\
R=0.000013 \bar{g}_{\mathrm{K}}+0.000205 \bar{g}_{\mathrm{K}(\mathrm{Ca})}-0.000016 \\
(n=19, \sigma=0.000075) . \quad(20)
\end{array}
$$

Where $n$ is the number of cells, and $\sigma$ is the standard deviation of the specific variations from the linear combination.

\section{The $\operatorname{MCS}(T)$ Function}

We define the function $\operatorname{MCS}(T)$, to be the minimal charge needed to shift the membrane potential, $V(T)$, above threshold, $V_{\text {Thresh }}(T)$ and consequently to evoke a spike at time $T$ during the cell cycle.

To quantify this charge, the mathematical model (1) to (16) reduces the cell to an electrical circuit. This 


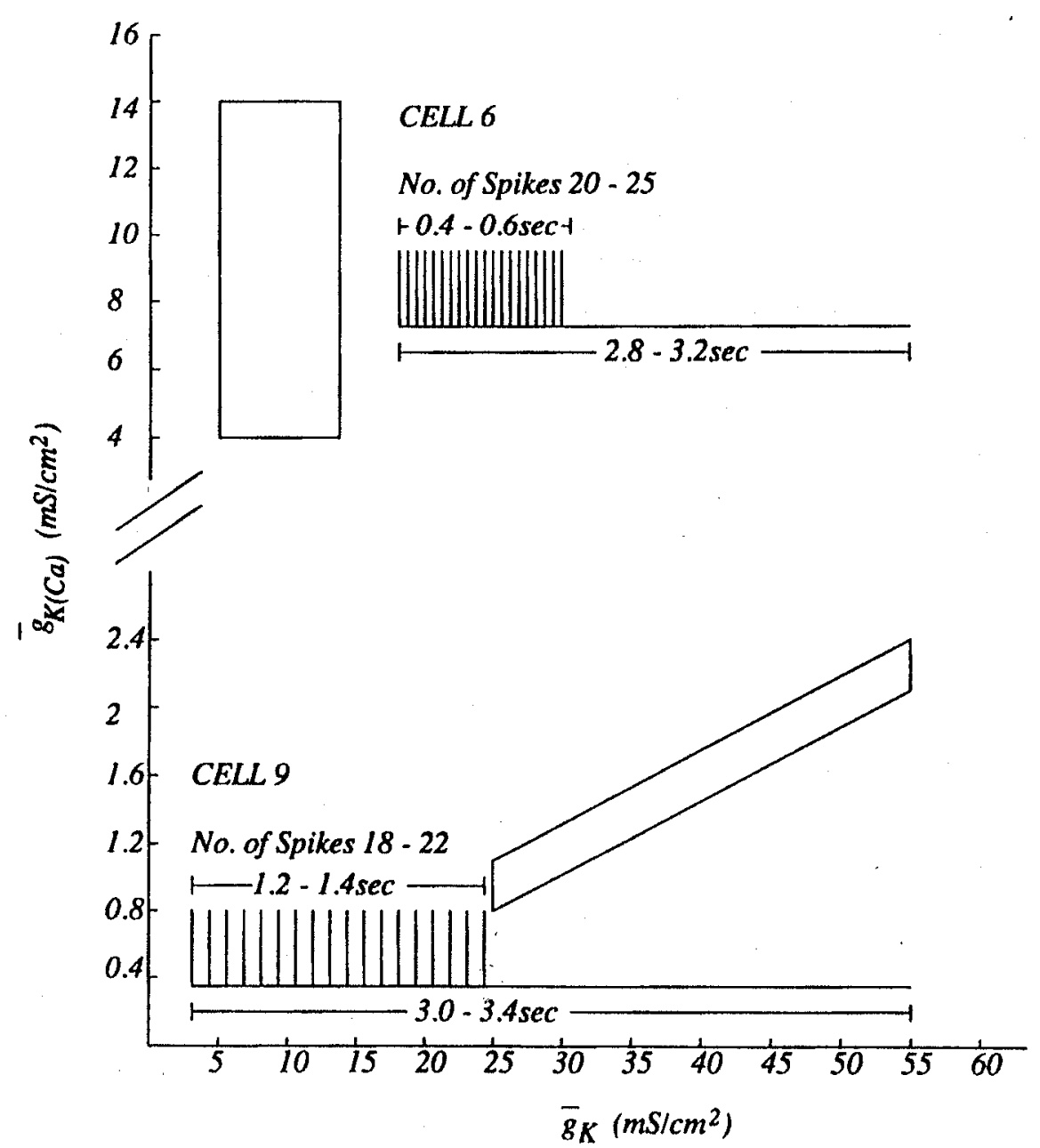

Figure 4. Correlation between burst characteristics and model parameters. The definition of bursters belonging to group- 6 is given in the upper schematic illustration. In order to obtain this kind of burster, the parameters $\bar{g}_{\mathrm{K}}$ and $\bar{g}_{\mathrm{K}(\mathrm{Ca})}$ must reside in a rectangle, where $5 \mathrm{mS} / \mathrm{cm}^{2}<$ $\bar{g}_{\mathrm{K}}<15 \mathrm{mS} / \mathrm{cm}^{2}$, and $4 \mathrm{mS} / \mathrm{cm}^{2}<\bar{g}_{\mathrm{K}(\mathrm{Ca})}<14 \mathrm{mS} / \mathrm{cm}^{2}$. The definition of bursters belonging to group-9 is given in the lower schematic illustration. For this kind of burster, the parameters $\bar{g}_{\mathrm{K}}$ and $\bar{g}_{\mathrm{K}(\mathrm{Ca})}$ must reside in a parallelogram, where $25 \mathrm{mS} / \mathrm{cm}^{2}<\bar{g}_{\mathrm{K}}<55 \mathrm{mS} / \mathrm{cm}^{2}$, and $0.032 \bar{g}_{\mathrm{K}}<\bar{g}_{\mathrm{K}(\mathrm{Ca})}<0.044 \bar{g}_{\mathrm{K}}$.

circuit contains a capacitor $C_{m}$, representing the membrane capacitance, and a variable conductor, $G_{\text {tot }}(V)$, representing the sum of the various channel conductances in the membrane. In such a circuit, the relation between charge, $Q$, and voltage, $V$, is given by

$$
Q=V \cdot C_{m}
$$

-In our case, the charge needed at time, $T$, to cause the desired shift in membrane potential is given by

$$
\operatorname{MCS}(T)=\left(V_{\text {Thresh }}(T)-V(T)\right) \cdot C_{m} .
$$

Numerical solution of the mathematical model (1) to (16) allows evaluation of $V(T)$, but not $V_{\text {Thresh }}(T)$.
The section below presents a procedure to determine $V_{\text {Thresh }}(T)$ numerically.

\subsection{Determining $V_{\text {Thresh }}(T)$}

To understand how $V_{\text {Thresh }}(T)$ can be determined, we begin with a phase plane analysis of the fast $V-W$ subsystem assuming the slow variables $X$ and $\mathrm{Ca}$ to have fixed values. Such a system can exhibit only two types of behavior. In the first type, endogenous oscillations, the cell fires continuously without any input. In the second type, the cell is quiescent and fires a spike only in response to an above-threshold 

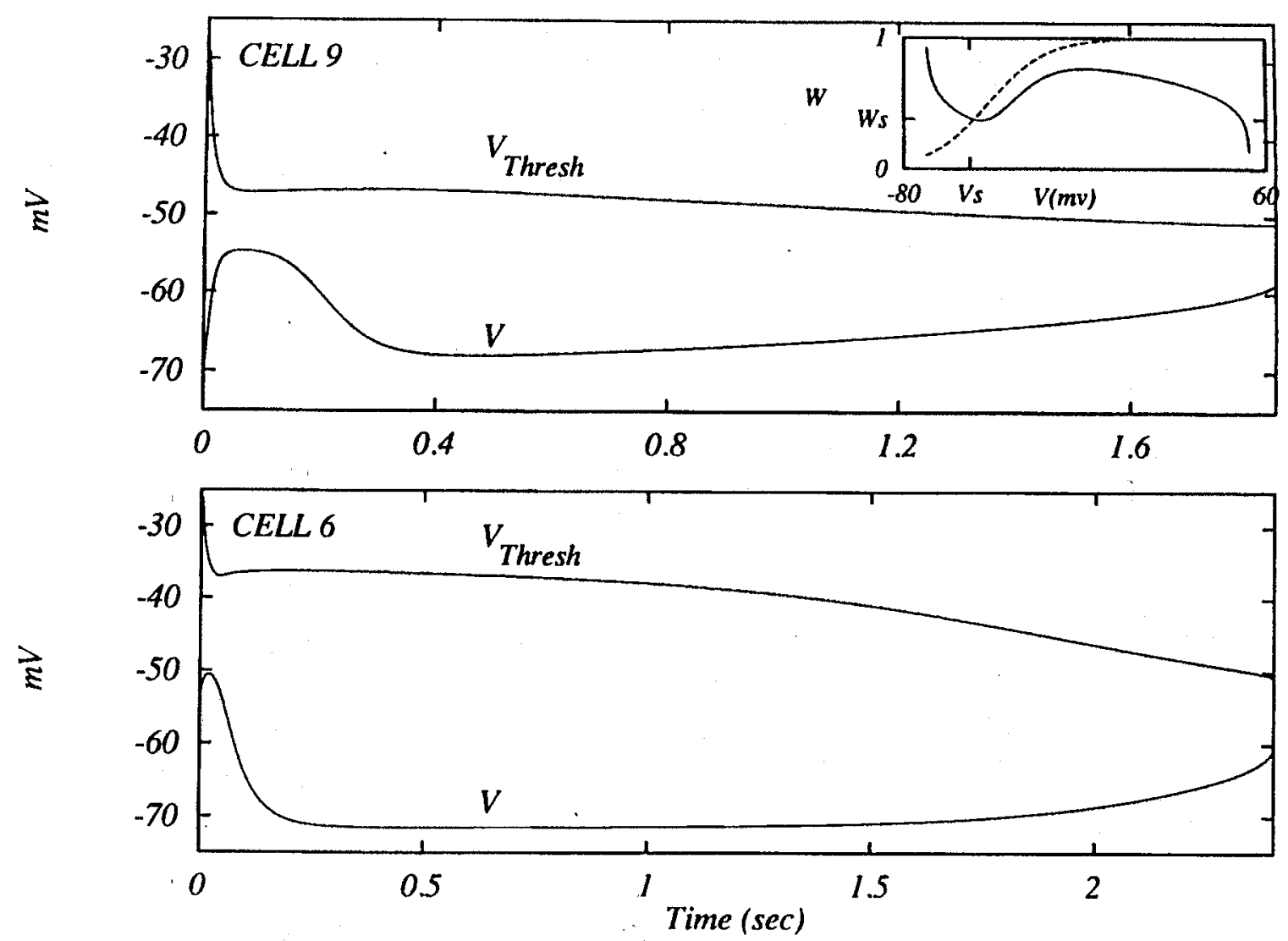

Figure 5. The threshold during the quiescent period. The threshold of cell 9 (top) and cell 6 (bottom) as calculated from the model, using the simplification that the $\mathrm{Ca}$ and $X$ variables can be regarded as constant when $V_{\text {Thresh }}$ is calculated. The membrane potential, $V(T)$, is shown for comparison. Inset: A typical $V$-nullcline and $W$-nullcline for fixed values of $\mathrm{Ca}$ and $X$. The two nullclines intersect in the falling portion of the $V$-nullcline, and therefore, the steady state $\left(V_{s}, W_{s}\right)$ is stable.

input (cells with this kind of behavior are often called followers).

In such follower cells, the $V$ and $W$ nullclines intersect once on the falling portion of the $V$ nullcline to give a stable steady state $\left(V_{s}, W_{s}\right)$ (see inset in Fig. 5). For any initial values of $V$ and $W$, the system will converge to the stable steady state $\left(V_{s}, W_{s}\right)$. For some initial values, however, the system will fire a single spike before reaching that stable state, while for others it will reach it without firing. In follower cells, $V_{\text {Thresh }}$ is defined as the smallest $V$ such that $V>V_{s}$, and the system generates a spike when the initial values are $\left(V, W_{s}\right)$.

In the model described here, both cell 6 and cell 9 show behavior much more complex than that of a follower cell. The system does not converge to a stable steady state but rather to a limit cycle that is, cyclic bursting behavior. An analysis of the kind undertaken for the follower cell cannot be performed unless the system is first simplified. Following Rinzel and Lee
(1987), we base our simplification on the observation that the model system can be divided into fast and slow processes. In fact, it is these two processes with very different time courses that generate the bursting behavior (Rinzel and Lee, 1987). The fast process, which includes $V$ and $W$, accounts for the spikes during the spiking period. The slow process, which includes $X$ and $\mathrm{Ca}$, shifts the cell back and forth between endogenous oscillations and the follower types of behavior.

Given that there are two time scales, a phase plane analysis for cells 6 and 9 can be done for the fast process, assuming the variables of the slow process $(\mathrm{Ca}(T)$ and $X(T))$ to be fixed. At each point in time $T$, we set the slow variables $\mathrm{Ca}$ and $X$ to be $\mathrm{Ca}(T)$ and $X(T)$ respectively and then calculated the stable state of the fast process, taking $X$ and $\mathrm{Ca}$ as constants. As expected, the fast process acquires a stable steady state throughout the quiescent period and so, one can calculate $V_{\text {Thresh }}(T)$ for the quiescent period (Fig. 5). 
Unlike a follower cell, where the threshold acquires a fixed value, the present model exhibits a time dependent threshold because the slow variables $\mathrm{Ca}$ and $X$ vary over the quiescent period.

\subsection{The Time Course of the MCS(T)}

We begin our discussion with the quiescent period; the spiking period will be discused later.
Figure 6A depicts the $\operatorname{MCS}(T)$ of cells 6 and 9, during the quiescent period, with $V(T)$ and $V_{\text {Thresh }}(T)$ evaluated as described above. At the beginning of this period, following the refractory period of the last spike, $V_{\text {Thresh }}(T)$ is still high (Fig. 5) but drops quickly as the $\mathrm{Na}$ and $\mathrm{K}$ channels recover. After this drop, $V(T)$ is still high (Fig. 5) due to the persisting influx of $\mathrm{Ca}$, which outlasts the last spike. Consequently, ( $V_{\text {Thresh }}(T)-V(T)$ ) is small (Fig. 6A). Once the $\mathrm{Ca}$ channels close (see $g_{\mathrm{Ca}}$ curve in Fig. 6B, 6C), $V(T)$
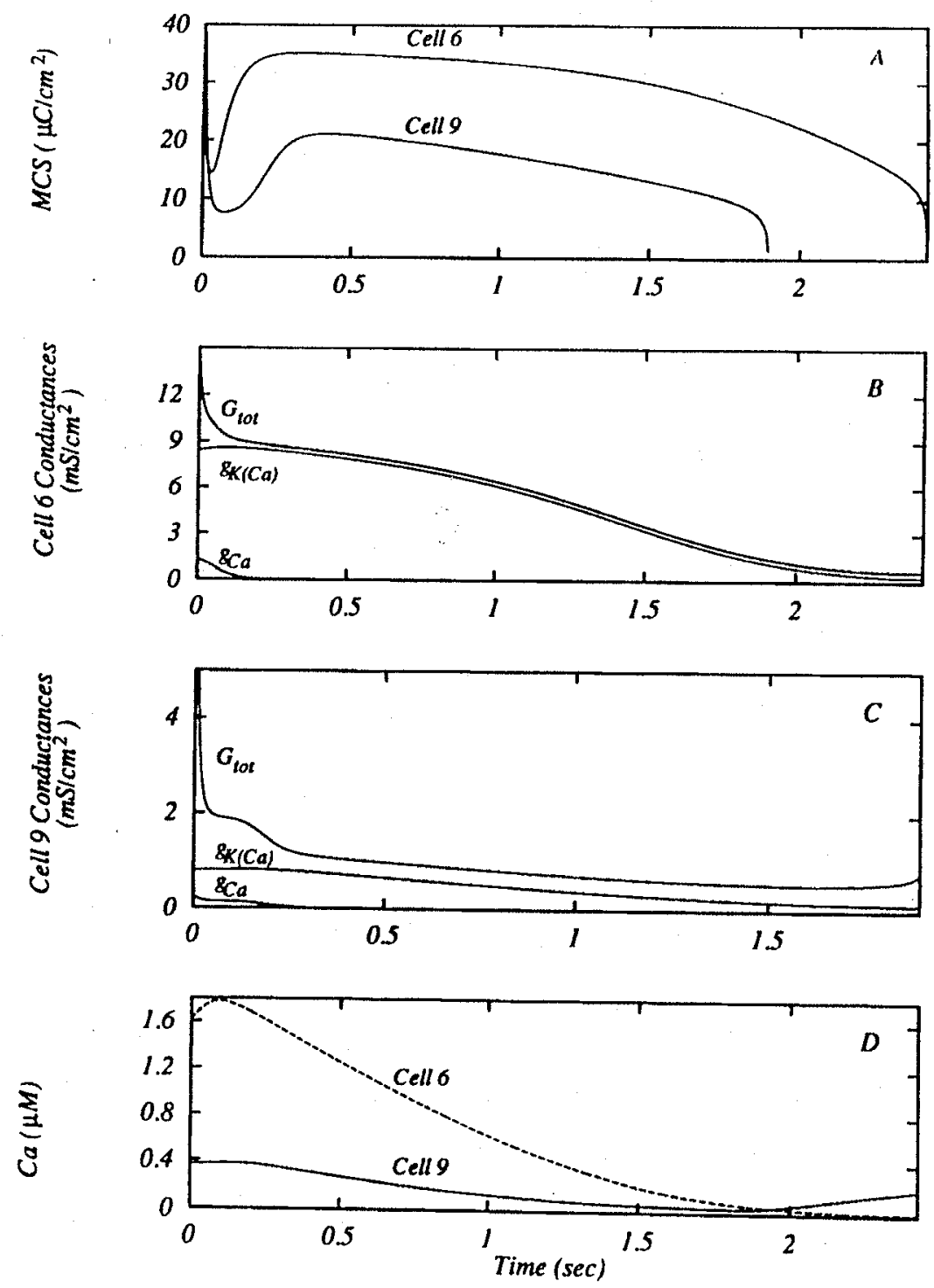

Figure 6. The parameters that control the $\operatorname{MCS}(T)$ shape. A: The $\operatorname{MCS}(T)$ of cells 6 and 9 . B: The conductances of cell 6 during the quiescent period. $G_{\mathrm{tot}}$ is the sum of all conductances. Not shown are $g_{\mathrm{K}}$ and $g_{\mathrm{Na}}$ because they are practically zero throughout the quiescent period. $g_{L}$, also not shown, is constant and $g_{L}=0.3 \mathrm{mS} / \mathrm{cm}^{2}$. C: Same as B but for cell 9. D: Ca concentration in cells 6 (dashed line) and 9 (solid line) during the quiescent period. Since the quiescent period of cell 9 is shorter than that of cell 6 , the last part of the solid line describes Ca concentration at the beginning of the spiking period of cell 9 . 
decreases but $V_{\text {Thresh }}(T)$ is still high due to the high concentration of $\mathrm{Ca}$ in the cell (Fig. 6D). This behavior leads to a rise in the $\operatorname{MCS}(T)$ (Fig. 6A). Later, when no more $\mathrm{Ca}$ enters, the removal of $\mathrm{Ca}$ becomes dominant (Fig. 6D); consequently, $V_{\text {Thresh }}(T)$ declines while $V(T)$ rises (both behaviors are due to the closing of calcium-dependent potassium channels (see $g_{K \text { (Ca) }}$ in Fig. 6B, 6C)). As a result, $\operatorname{MCS}(T)$ declines, thus acquiring a bell shape.

The observed difference in the amplitude of the $\operatorname{MCS}(T)$ of cells 6 and 9 (Fig. 6A) is due to the higher concentration of $\mathrm{Ca}$ in cell 6 (Fig. 6D). $V(T)$ is lower in this cell because more calcium-dependent potassium channels are open. The threshold in cell 6 is higher for the same reason (notice that at the end of the quiescent period when Ca concentration in both cells is the same, their thresholds coincide).

\section{The $\operatorname{MCPS}\left(T, t_{p}\right)$ Function}

The $\operatorname{MCS}(T)$ described above highlights the key underlying mechanisms that determine the sensitivity of cells 6 and 9 to an input pulse where the pulse duration, $t_{p}$, approaches zero. To extend the above discussion to longer pulses, we must consider the membrane conductances, $G_{\text {tot }}(T)$, in addition to the previously considered $V$ and $C_{m}$. We, therefore, define the function $\operatorname{MCPS}\left(T, t_{p}\right)$ to be the minimal charge of a brief current pulse needed to evoke a spike at time $T$. As an extension of (22), $\operatorname{MCPS}\left(T, t_{p}\right)$ is described by

$$
\operatorname{MCPS}\left(T, t_{p}\right)=\frac{\left(V_{\mathrm{Thresh}}(T)-V(T)\right) G_{\mathrm{tot}}(T)}{\left(1-e^{-t_{p} \cdot G_{\mathrm{tot}}(T) / C_{m}}\right)} \cdot t_{p}
$$

Indeed, when $t_{p} \rightarrow 0, \operatorname{MCPS}\left(T, t_{p}\right) \rightarrow \operatorname{MCS}(T)$.

In the context of (1) to (16) $G_{\text {tot }}(T)$ at any membrane potential is

$$
\begin{aligned}
G_{\mathrm{tot}}(T)= & \underbrace{\bar{g}_{\mathrm{Na}} m_{\infty}^{3}(V)(1-W)}_{g_{\mathrm{Na}}}+\underbrace{\bar{g}_{\mathrm{K}}\left(\frac{W}{s}\right)^{4}}_{g_{\mathrm{K}}}+\underbrace{\bar{g}_{L}}_{g_{L}} \\
& +\underbrace{\bar{g}_{\mathrm{K}(\mathrm{Ca})} \frac{\mathrm{Ca}}{\mathrm{K}_{d l}+\mathrm{Ca}}}_{g_{\mathrm{K}(\mathrm{Ca})}}+\underbrace{\bar{g}_{\mathrm{Ca} X}}_{g_{\mathrm{Ca}}}
\end{aligned}
$$

Figure 7 depicts the $\operatorname{MCPS}\left(T, t_{p}\right)$ for cell 6 and cell 9 for a brief pulse of $t_{p}=2 \mathrm{msec}$. The difference in the $\operatorname{MCPS}\left(T, t_{p}\right)$ of cells 6 and 9 is much larger than the difference in their corresponding $\operatorname{MCS}(T)$. This is because the denominator of (23) approaches 1 when $t_{p}$ does not approach zero and yet is short $\left(t_{p}>1 \mathrm{msec}\right)$. Under such conditions, the only difference between $\operatorname{MCS}(T)(22)$ and $\operatorname{MCPS}\left(T, t_{p}\right)(23)$ is that $G_{\text {tot }}$ scales the latter. Thus, the much higher $G_{\text {tot }}$ in cell 6 (compare $G_{\text {tot }}$ in Fig. 6B, 6C) is the main reason for the higher $\operatorname{MCPS}\left(T, t_{p}\right)$ in cell 6 .

Throughout the quiescent period, $G_{\text {tot }}$ is dominated by $g_{\mathrm{K}(\mathrm{Ca})}$ (Fig. 6B, 6C). Since $\bar{g}_{\mathrm{K}(\mathrm{Ca})}$ in group-9 is between $0.8 \mathrm{mS} / \mathrm{cm}^{2}$ and $2 \mathrm{mS} / \mathrm{cm}^{2}$ while in group-6 much higher and is between $4 \mathrm{mS} / \mathrm{cm}^{2}$ and $14 \mathrm{mS} / \mathrm{cm}^{2}$ (Fig. 4), it follows that the $\operatorname{MCPS}\left(T, t_{p}\right)$ in group-6 is always higher than that in group-9.

Two approximations were made in (23). First, we assumed that $G_{\text {tot }}(T)$ is constant during the pulse. Secondly, in calculating $V_{\text {Thresh }}(T)$ (Section 5.1), we assumed that no external current is applied. This obviously does not hold in the present case. To appreciate the magnitude of error introduced by these two approximations, we compare the behavior of $\operatorname{MCPS}\left(T, t_{p}\right)$ of (23) to a numerically simulated solution.

Equations (1) to (16) were solved numerically and a pulse current, $I_{\text {app }}$, was applied at various times, $T$, during the cell cycle. The duration of the pulse was fixed at $t_{p}=2 \mathrm{msec}$ and the minimal amplitude was set so as to evoke a spike at time $T$. This amplitude was then multiplied by the duration $t_{p}$ in order to obtain the charge. The results are depicted in Fig. 7 (inset); the $\operatorname{MCPS}\left(T, t_{p}\right)$ obtained from (23) (solid line) and from the numerical simulations (dashed line) exhibit similar behavior but differ in amplitude. The difference between the two is more pronounced in cell 6 (compare middle and top in inset to Fig. 7).

To understand the origin of these differences, we return to the approximations made in the solution of (23). We found that the first approximation, constant $G_{\text {tot }}(T)$, was quite accurate. The observed discrepancy must, therefore, be attributed to the second approximation. Indeed, phase plane analysis of $V$ and $W$ reveals that $V_{\text {Thresh }}$ decreases as $I_{\text {app }}$ increases. Since the required $I_{\text {app }}$ in cell 6 is much higher than in cell 9, the observed differences between the two $\operatorname{MCPS}\left(T, t_{p}\right)$ are larger in cell 6. As the pulse duration approaches zero, the distorting effect is diminished (bottom, Fig. 7 inset). With such a brief pulse, $V_{\text {Thresh }}$ decreases momentarily as $I_{\text {app }}$ is applied but soon returns to its original value (where $I_{\text {app }}=0$ ).

With the aid of the numerical simulation we could evaluate the $\operatorname{MCPS}\left(T, t_{p}\right)$ during the spiking period. 


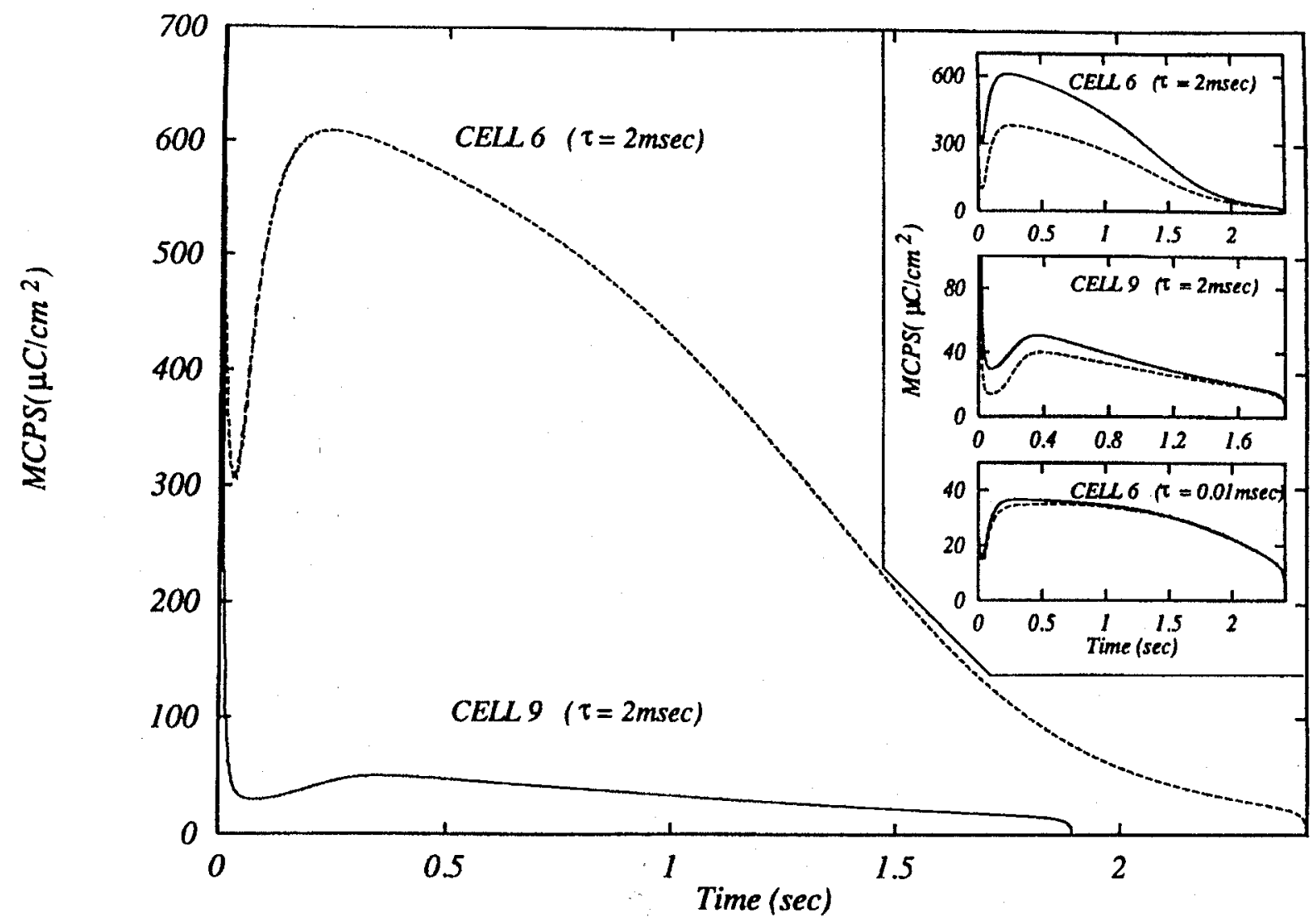

Figure 7. The MCPS $\left(T, t_{p}\right)$ of cells 6 and 9. The curves are solutions of (23) with $t_{p}=2$ msec. Inset: Numerical $((1)$ to $(16))$ versus the analytical (23) $\operatorname{MCPS}\left(T, t_{p}\right)$. In all cases solid line shows analytical solution and dashed line numerical one. Notice that when $t_{p} \rightarrow 0$ $\left(t_{p}=0.01 \mathrm{msec}\right.$, bottom) the two solutions coincide.

We found that, as expected, its value is high immediately after a spike and declines to zero before the next spike commences.

\section{Absolute and Relative Refractory Periods of a Burst}

We now examine the effect of an input current pulse on the intrinsic behavior of the cells' burst that is, cycle duration, burst duration, and number of spikes. The amplitude of the applied current must naturally, at least, equal the $\operatorname{MCPS}\left(T, t_{p}\right)$ amplitude to evoke a spike. Hence, a brief pulse of $2 \mathrm{msec}$ with an $\operatorname{MCPS}\left(T, t_{p}\right)$ amplitude (solution of (1) to (16)) was applied at each time $T$. The effect of this current (denoted MCPSamplitude current) on the above mentioned burst properties was examined.

The results for both cells 6 and 9 are shown in Fig. 8. In Fig. 8E, we plotted the $\operatorname{MCPS}\left(T, t_{p}\right)$ graph and divided it into three periods in which the applied MCPS-amplitude current exhibits a distinct effect.

The effect of the applied MCPS-amplitude current in the first period is exemplified in Fig. 8B by application of current at $T=0.5 \mathrm{sec}$ (that is, $0.5 \mathrm{sec}$ from the beginning of the quiescent period). As shown, the burst characteristics of both cells are almost unchanged (compare Fig. 8B to the control, Fig. 8A). During this period, which lasts about three-quarters of the quiescent period, the only effect of the applied current is to generate a single spike, and this increases the total cycle duration by $50 \mathrm{msec}$.

The second period corresponds to the last onequarter of the quiescent period (exemplified in Fig. 8C by an application of current at $T=2 \mathrm{sec}$ in cell 6 and $T=1.6 \mathrm{sec}$ in cell 9). During this period, the applied current causes the spiking period to start earlier, in fact to just follow the $\operatorname{MCPS}\left(T, t_{p}\right)$ "evoked" spike (compare Fig. 8C to the control, Fig. 8A). 
CELL 6
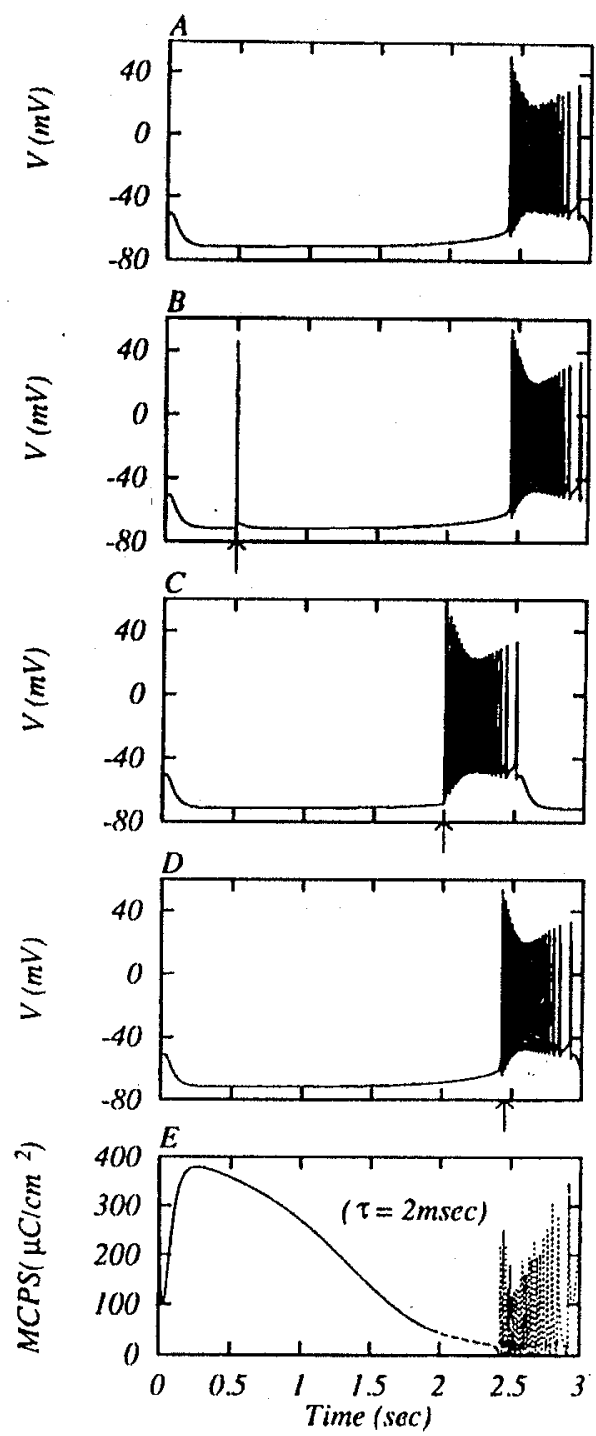

CELL. 9
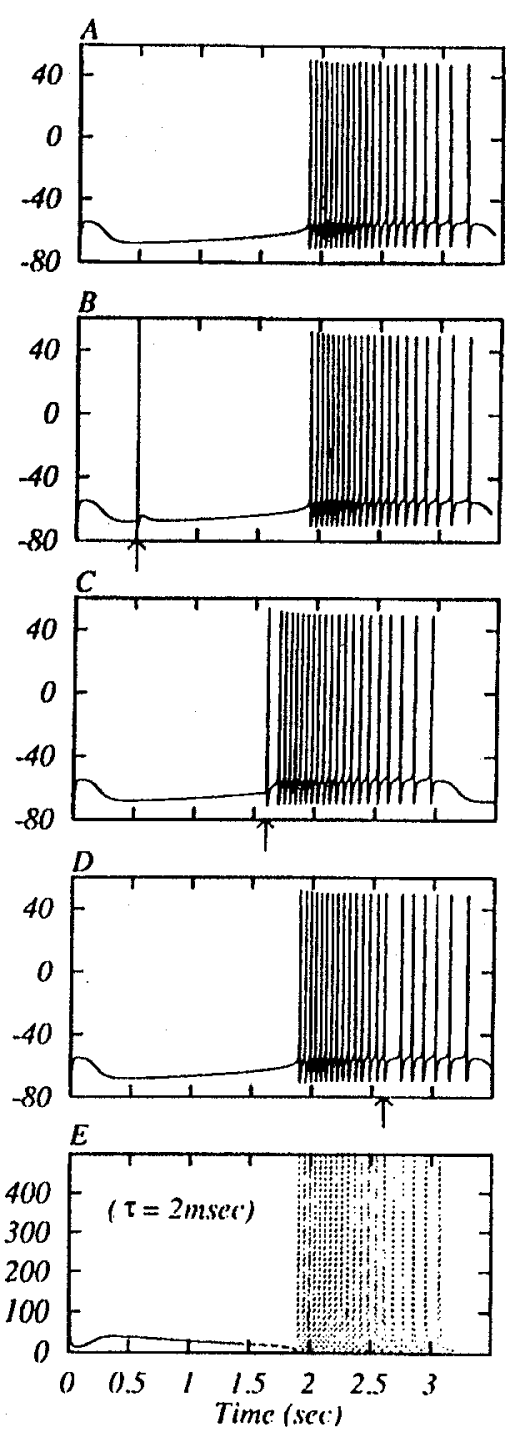

Figure 8. Effect of MCPS-amplitude current on the behavior of cells 6 and 9. For both cells, A: The "control" temporal pattern of membrane potential. B: $I=180 \mu \mathrm{A} / \mathrm{cm}^{2}, T=0.5 \mathrm{sec}$ for cell 6 and $I=20 \mu \mathrm{A} / \mathrm{cm}^{2}, T=0.5 \mathrm{sec}$ for cell 9. (note arrows). C: $I=23 \mu \mathrm{A} / \mathrm{cm}^{2}, T=$ $2 \mathrm{sec}$ for cell 6 and $I=10 \mu \mathrm{A} / \mathrm{cm}^{2}, T=1.6 \mathrm{sec}$ for cell 9 (note arrows). D: $I=9 \mu \mathrm{A} / \mathrm{cm}^{2}, T=2.45 \mathrm{sec}$ for cell 6 and $I=76 \mu \mathrm{A} / \mathrm{cm}^{2}$, $T=2.6 \mathrm{sec}$ for cell 9 . (note arrows). E: The $\operatorname{MCPS}\left(T, t_{p}\right)$ divided into three regions; solid line for the first period, dashed for the second, and dotted for the third (see text). In all applications $t_{p}=2 \mathrm{msec}$.

The effect is minimal when the current is applied during the spiking period (dotted line in Fig. 8E) (compare Fig. 8D to the control, Fig. 8A). The "evoked" spike is sometimes added to the total number of spikes in the burst and sometimes "replaces" one of the original spikes, such that the total number of spikes in the burst remains unchanged. In both cases, the overall behavior of the burst remains the same.
Figure 8 shows that the two cells exhibit a very similar behavior. Of particular interest is their bimodal behavior during the quiescent period. The first period (solid line in Fig. 8E), where the applied current cannot "evoke" a burst, can be looked upon, as the burst analog of the absolute refractory period following a single spike. Hence, we denote it as burst absolute refractory period (BAR). We denote the second period 
(dashed line in Fig. 8E) burst relative refractory period (BRR). MCPS-amplitude current applied during this period initiates the natural spiking period.

We next examined, at each time, $T$, during the internal cycle of the cell, whether application of currents with amplitudes higher or lower than the MCPSamplitude current generate effects different from those generated by applying the MCPS-amplitude current. When we applied current with one-half the amplitude of the MCPS current, there was no distinguishable effect on the cells' behavior. Upon application of a current with an amplitude twice as high as the MCPS current, no additional effect, beyond the effect of the MCPS current, was observed. We conclude that the $\operatorname{MCPS}\left(T, t_{p}\right)$ is a sufficient criterion for assessing the sensitivity of the cells to a brief input current.

\section{Mathematical Insight into the Burst Refractory Periods}

An important observation made in the preceding paragraph concerns the unexpected bimodal effect of applying an MCPS-amplitude current during the quiescent period. For about three-quarters of the period (BAR), only a single spike is evoked, whereas an MCPS current during the last one-quarter, (BRR), evokes a full spiking period.

To highlight, mathematically, the underlying causes of the bimodal response, we treat the model as including both a fast and a slow process, each occurring on a different time scale. As discussed earlier, the slow variables, $\mathrm{Ca}$ (the intracellular calcium concentration) and $X$ (the fraction of open calcium channels) are responsible for the transition from a quiescent to a spiking period. As can be seen for cell 6 (Fig. 9A), spiking begins when $\mathrm{Ca}$ and $X$ shift the fast system into an unstable region (dashed lines). Thus, to understand why the spiking period starts earlier, we must focus on the effect of the $\operatorname{MCPS}\left(T, t_{p}\right)$ evoked spike on Ca and $X$.

We have found (not shown) that throughout the quiescent period, the $\operatorname{MCPS}\left(T, t_{p}\right)$, together with the evoked spike have a fairly constant effect on both slow variables, with a more significant change in $X . X$ increases by a constant $\triangle X(0.1$ in cell 6$)$ and Ca by a constant $\triangle C(0.01$ in cell 6$)$. Figure $9 \mathrm{~B}$ shows that when the $\operatorname{MCPS}\left(T, t_{p}\right)$ is applied no earlier than $T=1.94$ sec (from the beginning of the quiescent period) the fast system crosses from a stable to an unstable region causing the spikes to begin earlier.
Sivan et al. (1995) have shown that the mathematical mechanism underlying bursting behavior differs between cells 6 and 9 . However, due to the resemblance between the BAR and BRR properties of the two cells, we believe that in cell 9 the changes in $X$ and $\mathrm{Ca}$ are also responsible for the earlier occurrence of spikes.

\section{Discussion}

We have explored the sensitivity of different burster types to an elementary input, i.e., a brief depolarizing pulse. The main results are as follows:

- We identified a domain of the relevant parameters $\left(\bar{g}_{\mathrm{K}}, \bar{g}_{\mathrm{K}(\mathrm{Ca})}, \bar{g}_{\mathrm{Ca}}, R\right)$ where variations of cell 6, group6 , are obtained and another domain of the same parameters where variations of cell 9 , group- 9 , exist.

- The MCPS $\left(T, t_{p}\right)$ is a good indicator of the sensitivity of cells to a brief input pulse. With a fixed $t_{p}$, at any time, $T$, application of currents smaller than the MCPS current hardly affect the cell's behavior, while higher currents have the same effect as the MCPS current.

- In all model cells, the magnitude of the $\operatorname{MCPS}\left(T, t_{p}\right)$ is closely linked to the characteristic bursting behavior of the cell. During the quiescent period, the $\operatorname{MCPS}\left(T, t_{p}\right)$ acquires a distinct bell shape. During the spiking period $\operatorname{MCPS}\left(T, t_{p}\right)$ magnitude correlates with the refractory period that follows a single spike that is, a very high amplitude immediately after the spike that decrease toward the commencement of the next spike.

- For $t_{p}>1$ msec the magnitude of the $\operatorname{MCPS}\left(T, t_{p}\right)$ during the quiescent period in group- 6 is much higher than that in group-9. This observation correlates with the value of $g_{\mathrm{K}}(\mathrm{Ca})$ which is up to 10 times higher in group- 6 than in group-9.

- The effects of the MCPS-amplitude current on the cell characteristics during the quiescent period is bimodal. BAR, burst absolute refractory period, lasts about three-quarters of the quiescent period. During BAR, the cell, apart from firing one additional spike in response to the current application, shows practically no change in its intrinsic behavior. BRR, the burst relative refractory period, begins just after BAR and lasts until the beginning of the intrinsic spiking period. During BRR, an application of current initiates the intrinsic spiking period with some variations. 

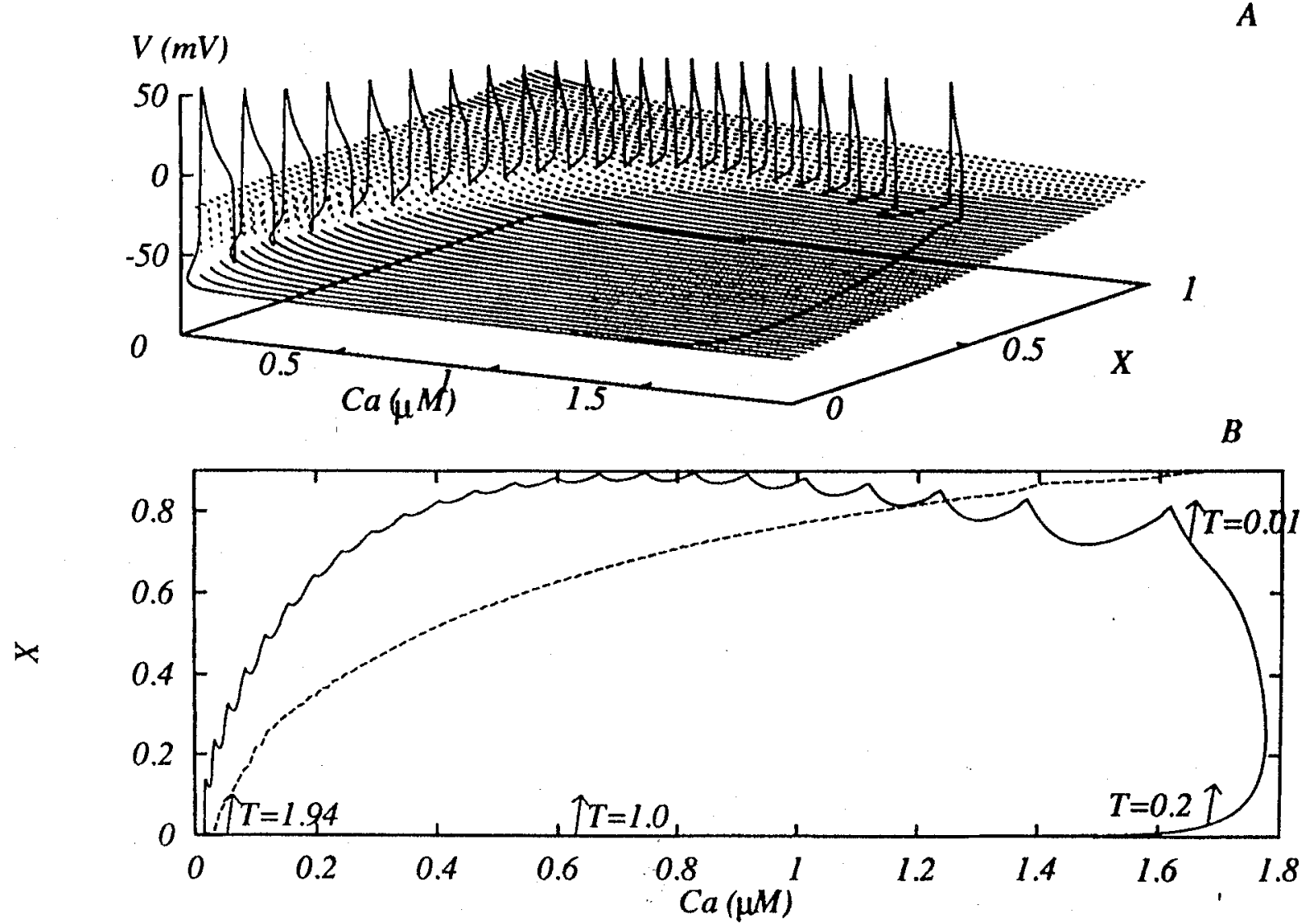

Figure 9. The fast system state during the cell cycle. A: Trajectory of $(\mathrm{Ca}, X, V)$ for cell 6 . Below the trajectory is a plane, each point of which describes the stability of the fast system if $\mathrm{Ca}$ and $X$ are frozen at their instantaneous values. The plane is dashed or solid depending on whether the steady state is unstable or stable. Note that spiking (except the two last spikes) takes place when the trajectory is in the unstable domain of the plane. (For the mechanism underlying the last two spikes, see Sivan et al., 1995). B: The trajectory of (Ca, $X$ ) for cell 6 (solid line). The dashed line divides the surface into a region where the fast system steady state is unstable (above the dashed line) and a region where the fast system steady state is stable (below). $T$ indicates the time from the beginning of the cycle. At each time, $T$, an arrow bar indicates a fixed $\triangle X(=0.1)$ and $\triangle C(=0.01)$ added to the $X$ and $C a$ values respectively at that time. This shows what would have been the new value of $X$ and $\mathrm{Ca}$ if the MCPS-amplitude current were applied at that time. Only at $T=1.94 \mathrm{sec}$ that is, in the last quarter of the quiescent period, is the fast system shifted to the unstable region.

These results are competible with results obtained from biological experiments. Tazaki and Cooke (1979) succeeded in recording from the small interneurons in the cardiac ganglion of the crab Portunus sanguinolentus but did not clearly identify from which cell recordings were made. These authors applied a brief input of $0.5 \mathrm{msec}$ at various time points during the quiescent period and observed the bimodality predicted from our model (Tazaki and Cooke, 1979, Fig. 14). In Mayeri's experiments (1973) the conditions were quite different both from those considered here and those employed by Tazaki and Cooke (1979); recordings were made from the entire ganglion, and the input was a brief electric shock. Under these conditions, the ganglion did not respond with a burst when the input was applied during the early part of the quiescent period, but a full burst was seen when the input was applied toward the end. When the electric shock was applied during the latter part of the burst, it terminated the burst. Mayeri (1973) also measured the threshold (minimal shock duration needed to evoke a burst) during the quiescent period. He found that from the time where a burst could be evoked, the threshold declined throughout the quiescent period. Mayeri's results to some extent 
resemble ours and those of Tazaki and Cooke (1979). The differences may result from the different experimental conditions.

The burst mechanism presented in our work partly falls into one of the categories discussed by Rinzel (1987). It relies on oscillations of the slow process which shifts the fast process from a firing to a nonfiring mode. This applies both for cell 6 and cell 9 . There is, however, an important difference in cell 9 (discussed in details in Sivan et al., 1995) as it fires when the fast process is in a stable steady state.

Bertram (1993a, b) has presented model similar to ours with respect to the two slow variables. He also noticed that reset of the burst is possible following a depolarizing perturbation in the quiescent period. Although our results are similar his interpretation is somewhat different from ours. Bertram (1993b) focused on the strength of the perturbation needed to terminate the quiescent period (he defines termination as the firing of even a single spike). In contrast, we distinguished between a period where only a single spike is evoked and that where resetting of the full burst occurs. The effect of perturbations on burst behavior was also discussed by Rinzel (1985). The case discussed there differs from ours as it concerned bistability as the underlying burst mechanism.

The long range goal of this study is to examine the thesis that the structure of a network that is, number of cells, connections between cells, and cell properties is based, at least in part, on considerations of fault tolerance. Our results indicate, how cells in the cardiac ganglion may, to some extent, tolerate erroneous behavior of other cells in the ganglion. First, during the BRR period, the endogenous bursters can become synchronized even if their internal cycle is not exactly the same. For example, cell 6 , with a longer intrinsic cycle than the rest of the interneurons (see Fig. 3), fires its spikes with the other cells because synaptic input onto cell 6 during its BRR period initiates its intrinsic spiking period.

The BAR is a very powerful tool for overcoming an erroneous input from a cell that may be firing irregularly at low frequency during its quiescent period, due to a high level of noise in the system or some intrinsic malfunctioning. The other cells do not change their intrinsic burst to follow this cell if these errors occur at the beginning of their quiescent periods. This may enable the system to maintain a sufficient interval between heart contractions.
Result 4 is of importance for yet another aspect of proper functioning of the cardiac ganglion. The $\operatorname{MCPS}\left(T, t_{p}\right)$ of cell 6 is very high, and it is practically impossible to make it fire even one spike during its BAR interval. Cell 6 is the activator of the motoneurons which activate the heart. As such, it must serve as an effective barrier against too frequent stimulation of the heart.

We have started our study with the most elementary input a brief input pulse. We plan to extend this study to include other inputs such as trains of brief pulses (representing inputs from oscillators or bursters) or prolonged pulses (representing hormonal effects).

\section{References}

Akaike N, Lee KS, Brown AM (1978) The calcium current of the Helix neuron. J. Gen. Physiol. 71:509-531.

Av-Ron E (1992) Ph.D. thesis Weizmann Institute. Rehovot, Israel.

Av-Ron E, Parnas H, Segel LA (1991) A minimal biophysical model for an excitable and oscillatory neuron. Biol. Cybern. 65:487-500.

Av-Ron E, Parnas H, Segel LA (1993) A basic biophysical model for bursting neurons. Biol. Cybern. 69:87-95.

Bertram R (1993a) A computational study of the effects of serotonin on a molluscan burster neuron. Biol. Cybern. 69:257-267.

Bertram R (1993b) Reduced-system analysis of the effects of serotonin on a molluscan burster neuron. Biol. Cybern. 70:359-368.

Friesen WO (1975a) Physiological anatomy and burst pattern in the cardiac ganglion of the spiny lobster Panulirus interuptus. $J$. Comp. Physiol. 101:173-189.

Friesen WO (1975b) Synaptic interaction in the cardiac ganglion of the spiny lobster Panulirus interuptus. J. Comp. Physiol. $10(1): 191-205$.

Hagiwara S, Takahashi K (1967) Surface density of calcium ions and calcium spikes in the Barnacle muscle fiber membrane. $J$. Gen. Physiol. 50:583-601.

Hartline DK (1967) Impulse identification and axon mapping of the nine neurons in the cardiac ganglion of the lobster, Homarus americanus. J. Exp. Biol. 47:327-340.

Hodgkin AL, Huxley AF (1952) A quantitative description of membrane current and its application to conduction and excitation in the nerve. J. Physiol. (London) 117:500-544.

Llinas R, Steinberg IZ, Walton K (1981) Relationship between presynaptic calcium current and postsynaptic potentiation in squid synapse. Biophys. J. 33:322-351.

Lustig C, Parnas H, Segel LA (1989) Neurotransmitter release: Development of a theory for total release based on kinetics. J. Theor. Biol. 136:151-170.

Mayeri E (1973) A relaxation oscillator description of the burstgeneration mechanism in the cardiac ganglion of the lobster, Homarus americanus. J. Gen. Physiol. 62:473-488.

Parnas H, Rudolph L (1991) Representation of synapses in neuronal networks. Technical Report, The Leibniz Center for Research in Computer Science, Hebrew University, Jerusalem, Israel. 
Parnas H, Segel LA (1989) Facilitation as a tool to study the entry of calcium and the mechanism of neurotransmitter release. Prog. Neur: (Kerkut GA, Phillis JW) 32:1-9.

Rinzel J (1984) Excitation dynamics: Insights from simplified membrane models. Proc. of 68th Annual Meeting of Fed. Am. Soc. Exp. Bio. St. Louis, MO.

Rinzel $J$ (1985) Bursting oscillations in an excitable membrane model. In: RJ Jarvis, BD Sleeman, eds. Ordinary and partial differential equations. Springer-Verlag, New York. pp. 304-316.

Rinzel J (1987) A formal classification of bursting mechanisms in excitable systems. In: E Teramoto, M Yamaguti, eds. Mathematical topics in population biology, morphogenesis and neurosciences. Lecture notes in biomathematics, Vol. 71, Springer, Heidelberg, New York. pp 267-281.

Rinzel J, Lee YS (1987) Dissection of a model for neuronal parabolic bursting. J. Math. Bio. 25:653-675.

Sivan E, Segel LA, Parnas H (1995) Modulated excitability: A new way to obtain bursting neurons. Biol. Cybern. 72:455-461.

Tazaki K, Cooke IM (1979) Spontaneous electrical activity and interaction of large and small cells in cardiac ganglion of the crab Portunus sanguinolentus. J. Neurophysiol. 42:975-999. 